\title{
Bifurcation and chaos in spin-valve pillars in a periodic applied magnetic field
}

\author{
S. Murugesh 调 and M. Lakshmanan讯 \\ ${ }^{1}$ Department of Physics \& Meteorology, IIT-Kharagpur, Kharagpur 721 302, India and \\ ${ }^{2}$ Centre for Nonlinear Dynamics, School of Physics, Bharathidasan University, Tiruchirapalli 620 024, India
}

(Dated: November 10, 2018)

\begin{abstract}
We study the bifurcation and chaos scenario of the macro-magnetization vector in a homogeneous nanoscale-ferromagnetic thin film of the type used in spin-valve pillars. The underlying dynamics is described by a generalized Landau-Lifshitz-Gilbert (LLG) equation. The LLG equation has an especially appealing form under a complex stereographic projection, wherein the qualitative equivalence of an applied field and a spin-current induced torque is transparent. Recently chaotic behavior of such a spin vector has been identified by Zhang and Li using a spin polarized current passing through the pillar of constant polarization direction and periodically varying magnitude, owing to the spin-transfer torque effect. In this paper we show that the same dynamical behavior can be achieved using a periodically varying applied magnetic field, in the presence of a constant DC magnetic field and constant spin current, which is technically much more feasible, and demonstrate numerically the chaotic dynamics in the system for an infinitely thin film. Further, it is noted that in the presence of a nonzero crystal anisotropy field chaotic dynamics occurs at much lower magnitudes of the spin-current and DC applied field.
\end{abstract}

Bolstered by the importance of Giant Magneto Resistance (GMR), a sequence of experimental and theoretical developments in the last few years on current induced switching of magnetization in nanoscale ferromagnets has thrown open several prospects in next generation magnetic memory devices. The direct role of spin polarized current, as against the traditional applied field, in controlling spin dynamics has brought in the possibility of new types of current-controlled memory devices and microwave resonators. The system under consideration is primarily a nanoscale spinvalve pillar structure, with one free ferromagnetic layer and another pinned layer separated by a nonferromagnetic conducting layer. The behavior of the dynamical quantity of interest, the magnetization field in the free layer, is well modeled by an extended Landau-Lifshitz equation with Gilbert damping, which is a fascinating nonlinear dynamical system. The free layer is usually assumed to be of single magnetic domain. Owing to the highly nonlinear nature of the LLG equation it is imperative to study the chaotic dynamical regime of the magnetization field. Indeed, several recent experiments have exclusively focused on chaos aspect of the system. In this article we have shown that a small applied periodically varying (AC) magnetic field, in the presence of a constant spincurrent and a steady applied magnetic field, can induce parametric regimes displaying a broad variety of dynamics and period doubling route to chaos. A numerical study of the effects of a non-

\footnotetext{
*Electronic address: murugesh@phy.iitkgp.ernet.in

†Electronic address: lakshman@cnld.bdu.ac.in
}

zero anisotropy field reveals chaotic dynamics at much lower magnitudes of the spin-current and applied DC field. This could be an important factor to consider in microwave resonator applications of spin-valve pillars.

\section{INTRODUCTION}

Following the success of GMR, the next major development in classical computer technology is expected to be through MRAMs (1; 2; 3). Apart from a manifold reduction in power consumption, being inherently nonvolatile in nature, they also bring in the prospect of computers that need not be rebooted. Understandably, fabrication of MRAMs has been a major thrust area of research in the last two decades. Typically, the memory unit consists of two nanoscale magnetic films separated by a spacer conductor/semiconductor medium and works on the principle of GMR. The imminent prospects in the recording media industry has prompted a breadth of development in the field. Besides the eminent role as a memory unit, the possibility of a single MRAM as a logical unit has also been proposed $(4 ;$; $)$. One notable technological hiccup in fabricating large MRAM grids is the extent to which the applied magnetic field can be localized. This imposes limitations on the efficiency with which an individual unit can be manipulated. The applied field required for the purpose is in most cases the Oersted field generated through electrical currents. A significant step forward, in bypassing the limitation on field localization, occurred when Slonczewski and Berger independently envisaged a more direct role for (spin polarized) current on magnetization(6; 7). They predicted that the angular momentum acquired by the spin polarized current can interact with the magnetization vector, and thus a suitable spin polarized current 
can possibly flip its direction. This phenomenon has been well established in a series of experiments in the last decade and referred to as the spin-torque effect in the literature $(8 ; 19 ; 10 ; 11 ; 12 ; 13 ; 14 ; 15)$. Interestingly, the effect has a simple semiclassical description in the form of an extended Landau-Lifshitz equation with Gilbert damping $(6 ; 7 ; 16)$. Several proposals have appeared in the last few years suggesting an increased role of the spin current and the associated torque in the eventually expected version of the MRAM.

One important assumption often made in most of these studies is that the magnetization in the film is homogeneous, or at least enough well defined that one can consider the film to be a mono-domain layer. This homogeneity assumption effectively nulls the Heisenberg interaction between neighboring spins, and allows one to treat the system as a single spin unit. As the size of the magnetic film is increased, this approximation is expected to fail. Indeed, chaotic behavior has been observed due to spin field inhomogeneity at lateral sizes of order $60-130 \mathrm{~nm}(17 ; 18)$. Besides, it is well realized experimentally that spin-transfer can induce microwave oscillations $(17 ; 19 ; 20 ; 21)$. The possible role of such current controlled microwave oscillators in the nanoscale has been well realized (17). For higher power levels that are practically desired it is natural to look at a series of such coupled spin-valve oscillators. Studies on synchronization of networks of such coupled nano-spin transfer oscillators, each one modeled on the extended LLG equation have been carried out both experimentally and numerically in an effort to enhance the emitted power $(22 ; 23 ; 24 ; 25)$.

Since the extended LLG equation is highly nonlinear in nature, a detailed study of the underlying nonlinear dynamics in spin-valve structures becomes inevitable. The stability diagram based on Melnikov theory in the space of the external field along the direction of anisotropy and the strength of spin torque due to a DC current was obtained in detail by Bertotti et. al., in (26). Following this, it was shown by Z. Li, Y. C. Li and S. Zhang that an applied alternating current can induce three broad types of dynamics, vis $-a-v i s$ Chaos, Multiply periodic and Periodic (27). Qualitatively similar features are also noted when the effects of nearest neighbor interactions are included in a long one dimensional ferromagnet with a spin torque term. Indeed the ferromagnetic chain exhibits both periodic and chaotic behavior in the presence of an applied AC spin current. (28). The multiply periodic behavior refers to the case where, with change in parameter, the system moves from periodic to multiply periodic behavior but not leading to chaos. It must be mentioned at this point that the last two types 'multiply periodic' and 'periodic' are also referred to in the literature as 'modification' and 'synchronization'(27; 29). The usage here is deliberate as the periods do not have a direct relation to the period of the applied magnetic field. It was also shown that in the space of the applied external field and the strength of the spin current the system exhibits chaotic behavior for a range of values within the boundary predicted by Melnikov theory (29).

The possibility of chaotic oscillations in monodomain single nanoscale spin-valve requires further in depth study of underlying bifurcation and chaos scenarios, including the detailed phase diagrams, at least for two reasons: (i) Chaotic oscillations in spin transfer oscillators may be unfavorable from a practical/technological point of view and such oscillations need to be suppressed by minimal intervention using one of the several controlling techniques developed in the field of chaotic dynamics (30; 31), where already such a study has been made (32). (ii) The previously mentioned possibility of synchronization of coupled spin-valve oscillators raises complex problems which are new in spintronics and is related to the topic of chaos synchronization in dynamical systems $(30 ; 31 ; 33)$, similar to synchronization of chaotically evolving networks of Josephson junctions, laser systems and nonlinear oscillators. As synchronization of chaotic oscillators is considered as a potential technique for secure communication including cryptography, there exists possible applications of networks of nanoscale spinvalve structures along these lines, although this may be complicated due to the presence of several parameters in the system. Consequently the study of the full nonlinear dynamics of spin transfer oscillators will be of considerable significance. It may be noted that chaos in magnetic systems, such as yttrium garnet, driven by external fields have been extensively studied in the past $(34 ; 35 ; 36)$. Numerical experiments on a model of thin magnetic film wherein spin wave excitations induced by spin-current lead to chaos have also been studied in (37). However, chaos in nano-spin valve geometry is reasonably new with potential new applications as discussed above.

In this paper, we study the chaotic dynamics of the magnetization vector in a single domain current driven spin-valve pillar, induced by a periodically varying $(\mathrm{AC})$ applied magnetic field in the presence of a constant spin current and steady (DC) magnetic field, using the extended LLG equation as the model for the system. Making use of a complex stereographic variable, we observe that the spin current induced torque is effectively equivalent to an applied magnetic field. Following this observation, we show numerically that a periodically alternating field can lead to chaotic behavior of the magnetization vector, which is similar to that of an alternating spin-current induced torque, studied in $\mathrm{Li}, \mathrm{Li}$, and Zhang (27). It will be shown that the order of magnitude of the applied alternating field required for chaotic motion is substantially lower, within practically achievable limits, compared to the alternating current magnitudes shown in $(27 ; 29)$. This is expected to be helpful in applications such as resonators, as an AC magnetic field is much more feasible practically than a $\mathrm{AC}$ spinpolarized current (although, when it comes to DC fields, the current induced DC Oersted fields are more cumbersome to produce in spin valve geometries compared to DC spin currents). We study the dynamics in an infi- 
nite thin film, both with a vanishing and non-vanishing anisotropy field. In the setup we shall consider the monodomain spin layer influenced by a DC spin-current, and both a DC and AC applied magnetic field. Although the applied magnetic field is qualitatively equivalent to a suitable spin-current(38), we employ both a DC spincurrent and applied magnetic field as the magnitudes at which chaos is observed is quite high, and hence difficult to achieve exclusively using either of the two. It may be noted that the chaotic dynamics studied here is induced by an $\mathrm{AC}$ applied magnetic field, and is phenomenologically different from the spin field inhomogeneity induced chaotic behavior that is observed in (17). Periodic dynamics is possible even in the absence of an alternating current, or field, as has been noticed in ferromagnetic films induced by a spin-current (19). Futher it has been reported therein that the current magnitudes at which periodic behavior is seen share a linear relation of negative slope with the oscillation frequencies. Our numerical results based on the extended LLG model are shown to be in agrement with these observations. Interestingly, in the presence of a non-zero in plane easy-axis crystal anisotropy field (taken along the $z$ direction), the chaotic dynamical regime is observed at much lower magnitudes of the DC applied field and spin-current. This could be an aspect to factor in while designing spin-valve based microwave resonators.

The paper is organized as follows. In Section 2, we detail the various interactions, including spin-transfer torque, that make up the extended LLG equation. Further, we introduce a complex stereographic variable equivalent to the macro-magnetization vector, and rewrite the LLG equation in this variable. As will be shown, this elucidates the role of the spin transfer torque as equivalent to an applied magnetic field. In Section 3 , we present our numerical results which demonstrate chaotic dynamics of the magnetization vector in the presence of a periodic applied field. Here we shall consider two cases, namely response in the presence and absence of a crystal anisotropy field. As will be noticed, the chaotic regimes in the two cases are significantly different. In Section 4 we conclude with a discussion on our results.

\section{THE EXTENDED LANDAU-LIFSHITZ EQUATION AND COMPLEX REPRESENTATION}

A typical spin-valve pillar used in most experiments is schematically shown in FIG 1. A current passing through this ferromagnet acquires a spin polarization in the $\hat{\mathbf{z}}$ direction. The thickness of the spacer conductor medium should be less than the spin diffusion length of the polarized current. The polarized current then passing through the free layer causes a change in the magnetization vector $\hat{\mathbf{S}}$, an effective torque referred to as the spin transfer torque. Interestingly, it has been realized that semiclassically the phenomenon can be described by an extension

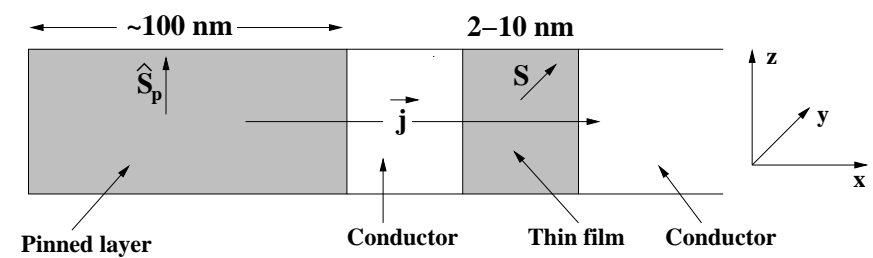

FIG. 1 A schematic figure of a spin-valve pillar. The cross section of the free layer is roughly $5000 \mathrm{~nm}^{2} . \hat{\mathbf{S}}$ is the magnetization vector in the free layer, and is the dynamical quantity of interest. $\hat{\mathbf{S}}_{P}$ is the direction of polarization of the spin current.

to the LLG equation, $(6 ;$; 7$)$

$$
\frac{d \hat{\mathbf{S}}}{d t}=-\gamma \hat{\mathbf{S}} \times \vec{H}_{e f f}+\lambda \hat{\mathbf{S}} \times \frac{d \hat{\mathbf{S}}}{d t}-\gamma a \hat{\mathbf{S}} \times\left(\hat{\mathbf{S}} \times \hat{\mathbf{S}}_{p}\right),
$$

Here, $\hat{\mathbf{S}}=\left\{S_{1}, S_{2}, S_{3}\right\}$ is the unit vector along the direction of the magnetization vector in the ferromagnetic film, which is the dynamical variable of interest, $\gamma$ the gyromagnetic ratio, and $\lambda$ the dissipation coefficient. $\vec{H}_{\text {eff }}$ in Eq. (1) is the effective field due to exchange interaction, anisotropy, demagnetization and an applied field (see (38) for details):

$$
\vec{H}_{e f f}=D S_{0} \nabla^{2} \hat{\mathbf{S}}+\kappa\left(\hat{\mathbf{S}} \cdot \hat{\mathbf{e}}_{\|}\right) \hat{\mathbf{e}}_{\|}+\vec{H}_{\text {demag }}+\vec{B}_{\text {applied }},
$$

$\hat{\mathbf{e}}_{\|}$being the unit vector along the direction of the anisotropy axis. The demagnetization field is obtained as a solution of the differential equation

$$
\nabla \cdot \vec{H}_{d e m a g}=-4 \pi S_{0} \nabla \cdot \hat{\mathbf{S}},
$$

and $\vec{B}_{\text {applied }}$ is the applied magnetic field on the sample. The last term in Eq. (10) is the additional term describing the spin-transfer torque, and the parameter ' $a$ ' depends linearly on the current density $j$. $\hat{\mathbf{S}}_{p}$ is the direction of magnetization of the polarizer, i.e., the polarization of the spin current.

In this study we shall assume the magnetization to be homogeneous. This effectively nulls the exchange interaction, while Eq. (3) can be immediately solved to give the demagnetization field as

$$
\vec{H}_{\text {demagnetization }}=-4 \pi S_{0}\left(N_{1} S_{1} \hat{\mathbf{x}}+N_{2} S_{2} \hat{\mathbf{y}}+N_{3} S_{3} \hat{\mathbf{z}}\right),
$$

where $N_{i}, i=1,2,3$ are conveniently chosen such that $N_{1}+N_{2}+N_{3}=1$ (after suitable rescaling of the magnitude of the spin). For a spherical sample $N_{1}=N_{2}=N_{3}$, and the demagnetization term is effectively null in Eq. (11). In the next sections we study chaotic dynamics shown by the system when an alternating magnetic field is applied.

Rewriting Eq. (1) using the complex stereographic variable $(39 ; 40)$

$$
\Omega \equiv \frac{S_{1}+i S_{2}}{1+S_{3}}
$$


provides a more comprehensible picture of the role of spin transfer torque. For the spin valve system, the direction of polarization of the spin-polarized current $\hat{\mathbf{S}}_{p}$ remains a constant, and lies in the plane of the film. Without loss of generality, we call this the direction $\hat{\mathbf{z}}$ in the internal spin space, i.e., $\hat{\mathbf{S}}_{p}=\hat{\mathbf{z}}$. As mentioned in Sec. 2, we disregard the exchange term. We choose the applied external field also in the $\hat{\mathbf{z}}$ direction, i.e., $\vec{B}_{\text {applied }}=\left\{0,0, h_{a 3}\right\}$. Defining

$$
\hat{\mathbf{e}}_{\|}=\left\{\sin \theta_{\|} \cos \phi_{\|}, \sin \theta_{\|} \sin \phi_{\|}, \cos \theta_{\|}\right\}
$$

and upon using Eq. (5) in Eq. (1), we get

$$
\begin{array}{r}
(1-i \lambda) \dot{\Omega}=-\gamma\left(a-i h_{a 3}\right) \Omega+i S_{\|} \kappa \gamma\left[\cos \theta_{\|} \Omega-\frac{1}{2} \sin \theta_{\|}\left(e^{i \phi_{\|}}-\Omega^{2} e^{-i \phi_{\|}}\right)\right]-\frac{i \gamma 4 \pi S_{0}}{\left(1+|\Omega|^{2}\right)}\left[N_{3}\left(1-|\Omega|^{2}\right) \Omega\right. \\
\left.-\frac{N_{1}}{2}\left(1-\Omega^{2}-|\Omega|^{2}\right) \Omega-\frac{N_{2}}{2}\left(1+\Omega^{2}-|\Omega|^{2}\right) \Omega-\frac{\left(N_{1}-N_{2}\right)}{2} \bar{\Omega}\right]
\end{array}
$$

where $S_{\|}=\hat{\mathbf{S}} \cdot \hat{\mathbf{e}}_{\|}$. Using Eq. (5) and Eq. (6), $S_{\|}$, and thus Eq. (7), can be written entirely in terms of $\Omega$. For further details of derivation of Eq. (7) see Ref. (38).

It is interesting to note that in this representation the spin-transfer torque, proportional to the parameter $a$, appears only in the first term in the right hand side of Eq. (77) as an addition to the applied magnetic field $h_{a 3}$ but with a prefactor $i$. Thus the spin torque term can be considered as an effective applied magnetic field (38). It was further explicitly shown in (38), in the absence of the crystal anisotropy field, that the switching time due to the spin-torque will be shorter by a factor $\lambda$, compared to that of magnetic field induced switching. Further, the spin-torque produced the dual effect of precession and dissipation even in the presence of the external applied field. The nature of switching of magnetization for other types of materials can be investigated by analyzing Eq. (77), and for typical materials this has been carried out in (38).

\section{CHAOTIC DYNAMICS}

Magnetization reversal in a spin mono-domain layer in the presence of both a steady applied magnetic field and a steady polarized current corresponds to a rather complicated phase diagram, as revealed in (26). Using Melnikov theory, it was also shown therein that the magnetization vector also has limit cycles for a range of values of the parameters, with frequency in the microwave range. The dynamical quantity in question, the magnetization vector $\hat{\mathbf{S}}$, is two dimensional, owing to its constant (unit) magnitude. Hence, chaotic behavior is ruled out. However, making the applied field, or current, time dependent is one way of increasing the effective dimensionality of the system to three and hence introduce a possibility of chaotic dynamics. Following (26) it has been shown in (27) that a small alternating current can produce a variety of dynamics, namely, Multiply periodic, Periodic and Chaos. It is also noticed that, along with a steady spin current of order 250 Oe and a steady applied magnetic field of the same order, inclusion of a small alternating spin polarized current leads to chaotic dynamics (29).

The dynamical similarity of the applied field and the spin-torque was noted in Section [I] In this section we show numerically that an applied AC field can also produce diverse dynamical scenarios and point out the advantages in using a periodically varying applied magnetic field instead of an alternating current. i.e., in Eq. (11) (or equivalently Eq. (7)) we take

$$
\vec{B}_{\text {applied }}=\left\{0,0, h_{a 3}+h_{a c} \cos \omega t\right\} .
$$

It will be noted that chaotic dynamics is possible at much lower DC applied field strengths and spin current in the presence of an anisotropy field.

For the film geometry, the film is taken to be in the $y-z$ plane, and the demagnetization field perpendicular to the film, the $\hat{\mathbf{x}}$ direction, i.e.,

$$
H_{\text {demagnetization }}=-4 \pi S_{0} S_{1} \hat{\mathbf{x}} .
$$

The saturation magnetization is taken to be that of permalloy, so that $4 \pi S_{0}=8400 O e$. Two different scenarios, one without anisotropy, and another with an in-plane easy axis anisotropy of strength $\kappa=45 O e$ along the $z$ direction, are investigated. All the numerical results that follow have been obtained by directly simulating Eq. (1) for the vector $\hat{\mathbf{S}}$. The same results were confirmed using Eq. (7) as well.

\section{A. Regions of Chaos in the presence of an applied alternating field}

As a first step we show below (FIG 2) the regions of chaos, or regions of positive Lyapunov exponent, in the space of the DC magnetic field and the DC current for an alternating applied magnetic field of magnitude $10 \mathrm{Oe}$ and frequency $15 \mathrm{~ns}^{-1}$, first without an anisotropy field, $\kappa=0$, (FIG 2a), and then with anisotropy field, 

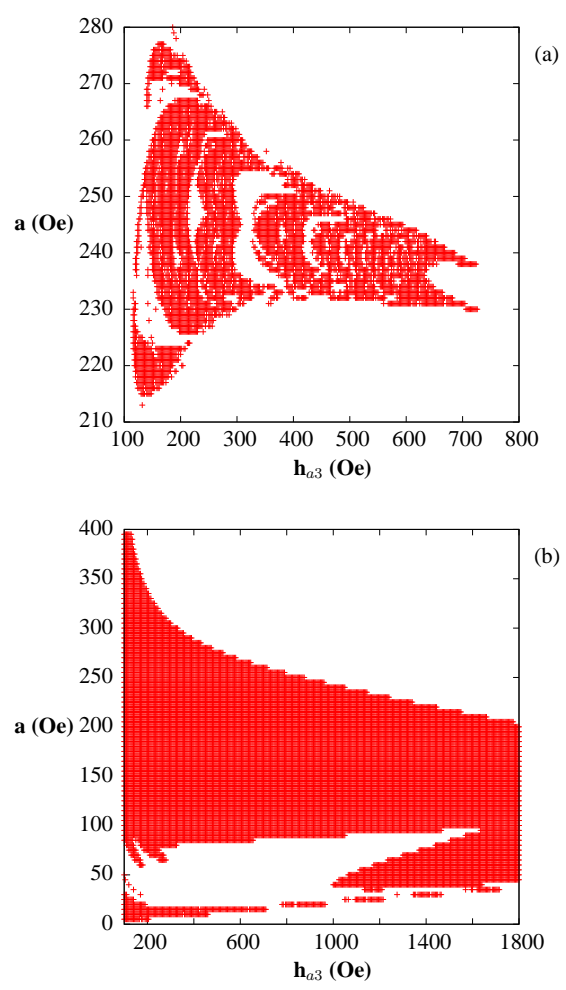

FIG. 2 Regions of chaos in the $a-h_{a 3}$ space, for an applied alternating magnetic field of amplitude $h_{a c}=10 \mathrm{Oe}$ and frequency $\omega=15 n s^{-1}$, a) without anisotropy field, $\kappa=0$, and b) with anisotropy field of strength $\kappa=45 \mathrm{Oe}$ along the $z$ direction. The dark regions indicate values for which the dynamics is chaotic, i.e, regions where the largest Lyapunov exponent is positive. In a) chaos is rarely noticed for lower values of $h_{a 3}$. The other parameters are $N_{1}=1, N_{2}=N_{3}=0$, $4 \pi S_{0}=8400 O e$. The points are plotted at intervals of $5 \mathrm{Oe}$ along both axes, and hence the figure offers only limited resolution in the dark(chaotic) regions.

$\kappa=45 O e$, (FIG 2b). The regions are obtained by direct numerical simulation using the model in Eq. (1), or equivalently Eq. (7). The dark regions in FIG 2 represent parameter values when the largest Lyapunov exponent is positive.

\section{Case a: No anisotropy $(\kappa=0)$}

The similarity of FIG 2a with that of regions of chaos for an alternating spin current must be noted (see FIG 1 in (29)). The figure is a demonstration of the qualitative equivalence of the applied field and current induced spintorque in describing the gross dynamical scenario. From FIG 2a, chaotic behavior of spins is observed for spincurrent magnitudes in the range of $a=200-300 \mathrm{Oe}$, and DC magnetic fields above $100 \mathrm{Oe}$. These values of ' $a$ ' correspond to spin-current magnitudes of over $10^{12} \mathrm{~A} / \mathrm{cm}^{2}$, which is at the higher end of the presently achievable levels.

In FIG 3 we present the bifurcation diagram showing the period doubling route to chaos as the DC current is varied. These diagrams are obtained by plotting the minimum values for one of the components of the spin, in this case $S_{1}$, over several periods of time for each value of $a$ in the given range. From the data in FIG 3a, the first five period doubling bifurcations are seen to occur at $a_{n}=268.4845,267.7723,267.6055,267.5685,267.5605$. Consequently, the ratio $\delta_{n}=\left(a_{n}-a_{n-1}\right) /\left(a_{n-1}-a_{n+1}\right)$ takes values $4.2698,4.5081,4.625$, clearly approaching the Feigenbaum constant. The Lyapunov spectrum for this range of ' $a$ ' is shown as inset. On comparison, we notice the largest Lyapunov exponent is positive for values of $a$ where the dynamics is chaotic in FIG 3a. A similar check has been made for FIG 3b, which again shows the period doubling route in the presence of an anisotropy field. Period doubling route to chaos is also noticed as the strength of the steady applied magnetic field is varied(keeping the current and frequency fixed), and when
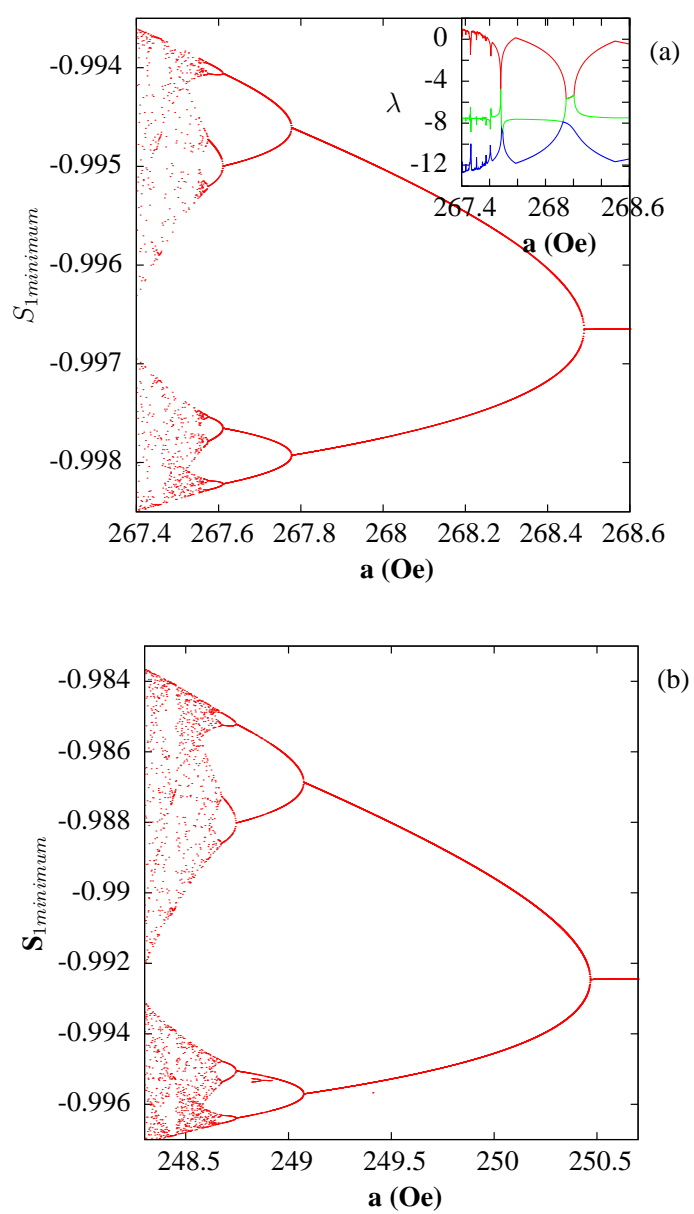

FIG. 3 Period doubling route to chaos as $a$ is varied. The figure is a plot of the minimum values of $S_{1}$ over several periods for the given parameter values a) without anisotropy, and $\mathrm{b}$ ) with anisotropy of $\kappa=45 \mathrm{Oe}$ along the $z$ direction. The applied DC field $h_{a 3}=200 \mathrm{Oe}$. All the other parameters remain the same as in FIG 2. The corresponding Lyapunov spectrum is shown as an inset. 
the frequency of the applied field is varied (keeping current and magnetic field strength steady), respectively.
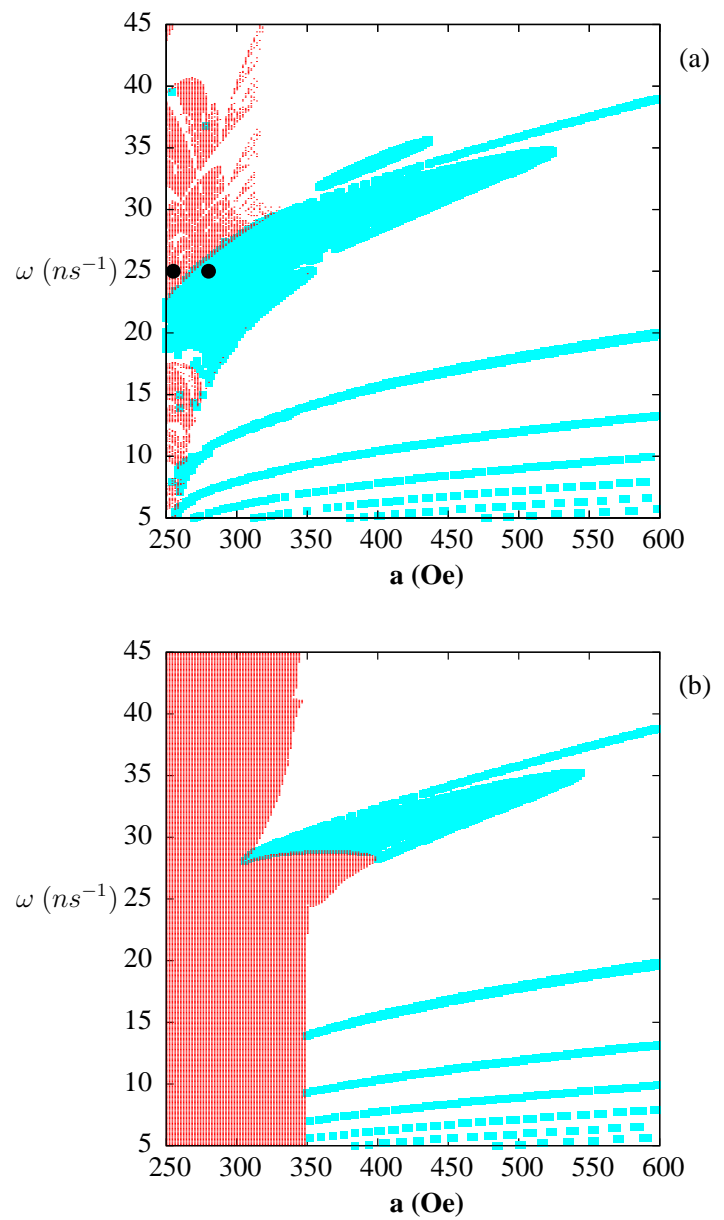

FIG. 4 Regions of chaos(red dots) and periodicity (blue wings) in the parameter space of DC current ' $a$ ' and frequency ' $\omega$ ', a) without anisotropy and b) with anisotropy ( $\kappa=45 O e)$ along the $z$ direction. The left over regions show multiply periodic behavior. All other parameters remain the same as in FIG 3. The power spectrum at the two dark points in (a) $(255,25)$ and $(280,25)$ are shown in FIG 5 (a) and (b), respectively.

\section{Case b: Non-zero anisotropy $(\kappa=45 \mathrm{Oe})$}

Contrary to the observation in FIG 2a, in the presence of a non-zero easy axis anisotropy, chaos is noted at much lower values of the spin current and DC applied field (FIG 2b). FIGs 3b shows period doubling bifurcation scenario in the presence of anisotropy. In FIG $3 \mathrm{~b}$ the current magnitude is varied, keeping the magnetic field strength and frequency of the $\mathrm{AC}$ component fixed. Similar period doubling route to chaos is also noticed as a) the magnitude of the steady magnetic field is varied (keeping current and frequency fixed), and when the frequency of the $\mathrm{AC}$ component of the applied mag- netic field is changed (keeping the current and magnetic field strengths constant). In either case, an easy axis anisotropy of magnitude $\kappa=45 \mathrm{Oe}$ is chosen along the $z$ direction, which is also the polarization direction of the spin current. The figures corresponding to these results are, however, not presented in here.

\section{B. Periodic, Multiply periodic and Chaotic dynamics}

In the presence of an $\mathrm{AC}$ spin-current induced torque, it is known that as the frequency of the spin-current is varied the system exhibits three distinct phases wherein the dynamics is predominantly either - Periodic, Multiply periodic or Chaotic(27). Herein we show that an applied AC magnetic field of magnitude $10 \mathrm{Oe}$, instead of a AC current, results in the three dynamical phases as the frequency of the applied field is varied for a constant value of the applied DC field.

FIG 4a shows regions of periodic (blue wings), and chaotic (red stem) dynamics in the parameter space of the spin current magnitude and the frequency of the applied magnetic field. Multiply periodic behavior is seen in the unshaded regions. Here again the similarity with that of FIG 1 in (27) may be noted. This further illustrates the qualitative similarity of the spin current induced torque with that of the applied field. Our numerical results further show a number of wing like bands in the $\omega-a$ space where periodic behavior is noticed. This is clearly absent with a periodic spin-current as seen in (27). The power spectrum corresponding to two specific points, one chaotic and the other periodic (indicated with dark dots in FIG 4a), are shown in FIG 5. Periodic behavior is noticed even in the limit $\omega \rightarrow 0$ for certain values of $a$, while multiply periodic behavior is noticed for the other intermediate values. The power spectrum corresponding to

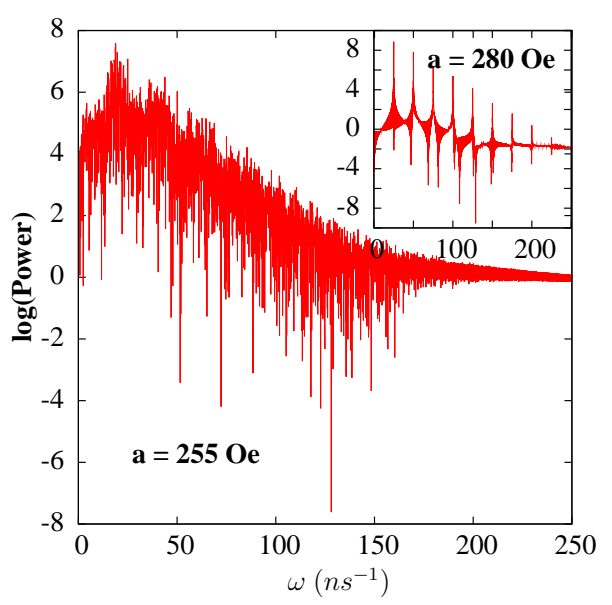

FIG. 5 The power spectrum distribution corresponding to periodic, $a=280 \mathrm{Oe}$ (inset), and chaotic, $a=255 \mathrm{Oe}$, scenarios in FIG 4a. The first peak in the inset is seen at $\omega=25 \mathrm{~ns}^{-1}$. The anisotropy is taken zero, and all other parameters are the same as in FIG 4a. 
these current values are shown in FIG. 6. Such a behavior, induced by a spin-current of constant magnitude, has been noticed in (19). Indeed, the current magnitudes, $a$, where periodic behavior is seen to vary linearly (with a negative slope) with the corresponding periods (see FIG. 6 inset), in further agreement with (19).

However, in the presence of the anisotropy field chaotic regime is much more pronounced and wider, as seen in FIG 4b. For a much lower value of the DC applied field $\left(h_{a 3}=20 O e\right)$ there appear wide bands in the $\omega-a$ space where periodic behavior is exclusively noted at low frequencies (FIG 7). As the frequency is increased multiple periodicity islands appear in between periodic bands, and for even higher frequencies the dynamics is largely one of multiply periodic type. No chaotic dynamics is noted in the parameter range chosen. These thick periodicity bands present better regions to choose in applications such as the microwave resonator discussed earlier, while chaos synchronization studies can be carried out in the chaos regimes.

\section{DISCUSSION AND CONCLUSION}

Using the complex stereographic variable to represent the spin vector, and rewriting the modified LandauLifshitz-Gilbert equation, we have shown that the spincurrent induced torque is qualitatively equivalent to an applied field. Using this equivalence we have shown that an applied AC magnetic field in the presence of constant spin current and DC applied magnetic field can lead to varied dynamical scenarios including chaos. We have explicitly demonstrated numerically the chaotic behavior for a range of values of the parameters. The sys-

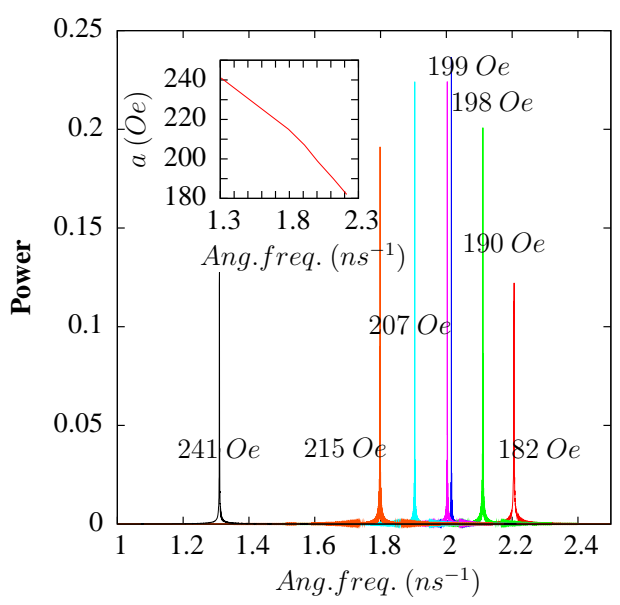

FIG. 6 The power spectrum distribution in the limit $\omega=0$, at certain values of $a$ (indicated on each spectrum) where periodic behavior is noted. Multiply periodic behavior is noticed for other values of $a$ in the range shown. The current magnitudes vary linearly and decrease with the frequency of oscillation (inset). $h_{a c}=0$, while all other parameters are the same as in FIG. 3.

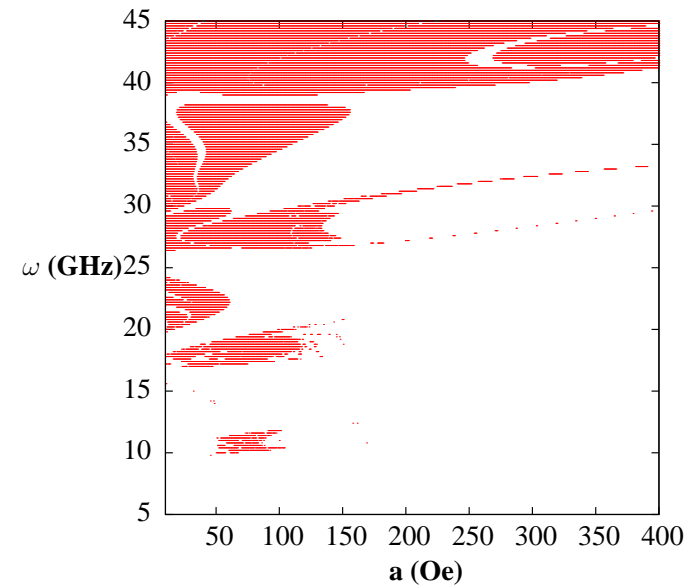

FIG. 7 Regions of multiply periodic dynamics for the system with the DC applied field fixed at $h_{a 3}=20 \mathrm{Oe}$, and non-zero anisotropy. All the other parameters remain the same as in FIG 4b. Synchronization is noted in the unshaded regions, while chaotic dynamics is not noticed in the parameter range shown in the figure. Islands of multiply periodic behavior appear between regions of periodic behavior for low frequencies. For higher frequencies, the dynamics is exclusively multiply periodic.

tem also exhibits regular periodic behavior for a different range of values. It is now realized that such nanoscale monodomain layers can find application as resonators, through periodic oscillations induced by an alternating spin-current. The results presented here provide an alternative method through oscillations induced by an applied magnetic field. It is further noticed that the range of the chaotic regime strongly depends on the presence of a crystal anisotropy field. In the presence of an anisotropy field chaotic behavior is noticed for much lower values of the DC field and spin-current, which are more suited for chaos synchronization studies. However, there are regions in the $\omega-a$ space where regular periodic motion is more robust and presents a better alternative in applications. In a future study we will present the possibility of chaos synchronization in spin-valve structures.

\section{ACKNOWLEDGMENTS}

S.M. wishes to thank DST, India, for funding through the FASTTRACK scheme. The work of M.L. forms part of a DST-IRHPA research project and is supported by a DST-Ramanna fellowship.

\section{References}

[1] S. A. Wolf, A. Y. Chtchelkanova and D. M. Treger, IBM J. Res. Dev. 50, 101 (2006).

[2] R. K. Nesbet, IBM J. Res. Dev. 42, 53 (1998). 
[3] C. H. Tsang, R. E. Fontana, Jr., T. Lin, D. E. Heim, B. A. Gurnet and M. L. Williams, IBM J. Res. Dev. 42, 103 (1998).

[4] W. C. Black, Jr. and B. Das, J. Appl. Ph. 87, 6674 (2000).

[5] A. Ney, C. Pampuch, R. Koch and K. H. Ploog, Nature 425, 485 (2003).

[6] L. Berger, Phys. Rev. B 54, 9353 (1996).

[7] J. C. Slonczewski, J. Mag. Mag. Mat. 159, L1 (1996).

[8] D. Berkov and J. Miltat, J. Mag. Mag. Mat. 320, 1238 (2008).

[9] M. D. Stiles and J. Miltat, Topics Appl. Physics 101, 225 (2006).

[10] E. B. Myers, D. C. Ralph, J. A. Katine, R. N. Louie and R. A. Buhrman, Science 185, 867 (1999).

[11] J.-E. Wegrowe, D. Kelly, X. Hoffer, Ph. Guittienne and J.-Ph. Ansermet, J. Appl. Phy. 89, 7127 (2001).

[12] J. A. Katine, F. J. Albert, R. A. Buhrman, E. B. Myers and D. C. Ralph, Phys. Rev. Lett. 84, 3149 (2000).

[13] J. Grollier, V. Cros, A. Hamzic, J. M. George and H. Jarrfés, Appl. Phys. Lett. 78, 3663 (2001).

[14] B. Özyilmaz, A. Kent, D. Monsma, J. Z. Sun, M. J. Rooks and R. H. Koch, Phys. Rev. Lett. 91, 067203 (2003).

[15] S. Urazhdin, Norman O. Birge, W. P. Pratt Jr. and J. Bass, Phys. Rev. Lett. 91, 146803 (2003).

[16] Y. B. Bazaliy, B. A. Jones and S.-C. Zhang, Phys. Rev. B 57, R3213 (1998).

[17] S. I. Kiselev, J. C. Sankey, I. N. Krivorotov, N. C. Emley, R. J. Schoelkopf, R. A. Buhrman, and D. C. Ralph, Nature 425, 380 (2003).

[18] K-J. Lee, A. Deac, O. Redon, J-P. Noziéres and B. Dieny, Nature Materials 3, 877 (2004).

[19] W. H. Rippard, M. R. Pufall, S. Kaka, S. E. Russek, and T. J. Silva, Phys. Rev. Lett. 92, 027201 (2004).

[20] W. Weber, S. Riesen, and H. G. Siegmann, Science 291, 1015 (2001).

[21] H. Xi, K. Z. Gao, and Y. Shi, Appl. Phys. Lett. 84, 4977 (2004).

[22] F. B. Mancoff, N. D. Rizzo, B. N. Engel and S. Tehrani,
Nature 437, 393 (2005)

[23] S. Kaka, M. R. Pufall, W. H. Rippard, T. J. Silva, S. E. Russek and J. A. Katine, Nature 437, 389 (2005).

[24] J. Grollier, V. Cros and A. Fert, Phys. Rev. B 73, 060409(R) (2006).

[25] J. Persson, Y. Zhou and J. Akerman, J. Appl. Phys. 101, 09A503 (2007).

[26] G. Bertotti, C. Serpico, I. D. Mayergoyz, A. Magni, M. d'Aquino, and R. Bonin, Phys. Rev. Lett. 94, 127206 (2005).

[27] Z. Li, Y. C. Li, and S. Zhang, Phys. Rev. B 74, 054417 (2006).

[28] Y. Lan, and M. C. Li, Nonlinearity 21, 2801 (2008).

[29] Z. Yang, S. Zhang, and Y. C. Li, Phys. Rev. Lett. 99, 134101 (2007).

[30] M. Lakshmanan and K. Muruali Chaos in Nonlinear Oscillators: Controlling and Synchronization (WorldScientific, Singapore, 1995).

[31] M. Lakshmanan and S. Rajasekhar, Nonlinear Dynamics (Springer-Verlag, Berlin, 2003).

[32] H. Z. Xu, X. Chen and J.-M. Liu, J. Appl. Phys. 104, 093919 (2008).

[33] A. Pikovsky, M. Rosenblum and J. Kurths, Synchronization: A Universal Concept in Nonlinear Sciences (Cambridge University Press, 2008).

[34] S. M. Rezende and F. M. de Aguiar, Pr. of IEEE. 78, 893 (1990).

[35] T. L. Carroll, M. L. Pecora and F. J. Rachford, J. Appl. Phys. 70, 3197 (1991).

[36] N. Y. Pisken and P. E. Wigen, J. Appl. Phys. 85, 4521 (1999).

[37] F. M. de Aguiar, A. Azevado and S. M. Rezende, J. Appl. Phys. 91, 8046 (2002).

[38] S. Murugesh, and M. Lakshmanan, Chaos, Sol. \& Frac. 41, 2773 (2009).

[39] C. Kosaka, K. Nakamura, S. Murugesh, and M. Lakshmanan, Physica D 203, 233 (2005).

[40] M. Lakshmanan and K. Nakamura, Phys. Rev. Lett. 53, 2497 (1984). 